\title{
Enfermedad de Hirschsprung
}

\section{Hirschsprung disease}

Santos-Jasso KA

\section{INTRODUCCIÓN}

El diagnóstico de la enfermedad de Hirschsprung se debe sospechar en el recién nacido que presenta datos de obstrucción intestinal: vómito, distensión abdominal y falla para el paso de meconio, en la primera semana de vida, lo que sustenta su origen congénito. Los pacientes con esta enfermedad son predominantemente del sexo masculino (84\%); suelen presentar impactación fecal colónica y en la valoración abdominal muestran desimpactación, que puede ser explosiva en el examen digital del recto con vaciamiento en el $96 \%$ de los pacientes. ${ }^{1}$ La progresión en cuanto a gravedad de estos síntomas suele manifestarse con fiebre, distensión abdominal, diarrea y sepsis; manifestaciones clínicas de una colitis obstructiva proliferativa, también Ilamada enterocolitis, la cual ocurre entre 11 a 24\% de los recién nacidos con enfermedad de Hirschsprung. ${ }^{2}$ Un subgrupo de pacientes con (aquellos que no presentan un cambio abrupto de calibre entre el segmento sano y el segmento afectado) manifiestan únicamente estreñimiento crónico, lo que dificulta el diagnóstico. ${ }^{1}$

Harald Hirschsprung describió una casuística de recién nacidos con estreñimiento secundario a la dilatación e hipertrofia del colon, sin afección aparente a nivel de sigmoides ni recto. ${ }^{3}$ Actualmente sabemos que el defecto de la migración neuronal intestinal céfalo-caudal traduce aganglionosis del recto en el $100 \%$ de los pacientes con ésta enfermedad; ${ }^{4}$ del sigmoides en $80-85 \%$; del colon descendente y colon transverso en $10 \%$ cada uno, y de colon ascendente y parte distal del íleon entre 5 y $10 \%$. Figura 1.
Cirujano Pediatra, Adscrita al Depto. de Cirugía.

Instituto Nacional de Pediatría, México.

Recibido: 28 de octubre del 2016

Aceptado: 17 de noviembre del 2016

Correspondencia

Karla Alejandra Santos Jasso Correo

santosjasso@hotmail.com

Este artículo debe citarse como

Santos-Jasso KA. Enfermedad de Hirschsprung. Acta Pediatr Mex. 2017;38(1):72-78. 


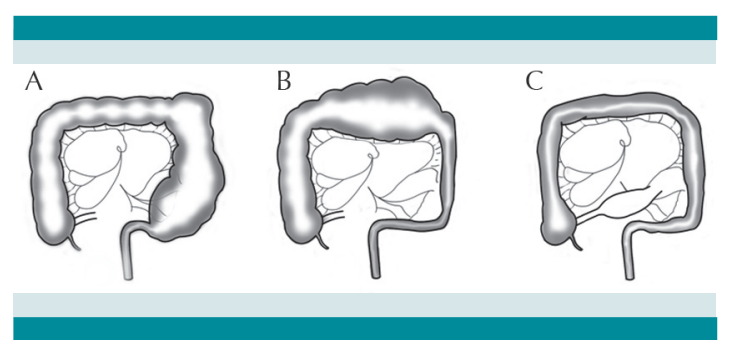

Figura 1. A) Enfermedad de Hirschsprung limitada al segmento de recto y sigmoides (80-85\% de los niños); B) Limitada al colon descendente, sigmoides y recto (10\%); C) Afecta al recto, colon con zona de transición en íleon terminal, próxima a la válvula ileocecal (5$10 \%$ ), en esta imagen podemos apreciar un colon sin pronunciamiento de las haustras.

El objetivo de este criterio pediátrico es describir las estrategias de diagnóstico y tratamiento de niños con enfermedad de Hirschsprung.

\section{DIAGNÓSTICO}

El estudio histopatológico de la biopsia del recto es el estándar de oro para realizar el diagnóstico (93\% sensibilidad, y $98 \%$ de especificidad), la cual idealmente debe ser tomada a $3 \mathrm{~cm}$ de la línea dentada. La ausencia de células ganglionares en el plexo submucoso con la tinción hematoxilina y eosina establece el diagnóstico. Figura 2. Sin embargo, en algunos casos es necesario realizar inmuno-histoquímica para células gangionales con proteína S-100 y calretinina para confirmar el diagnóstico. ${ }^{5-7}$ Figura 3.

La expresión clínica es la ausencia de relajación involuntaria del esfínter anal interno y puede ser registrada a través de una manometría anorrectal (sensibilidad de $91 \%$ y especificidad de $94 \%$ ). ${ }^{8}$

La manometría anorrectal es una prueba no invasiva para evaluar la función del esfínter anal interno. Esta prueba se realiza sin sedación, con el paciente en decúbito lateral izquierdo con las caderas y rodillas flexionadas y se introduce un

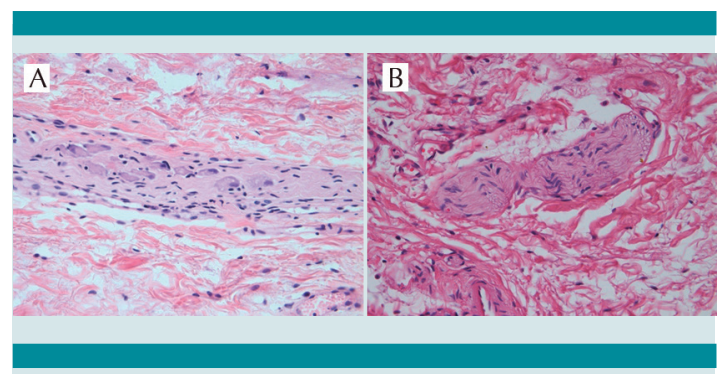

Figura 2. Cortes histológicos de plexos submucosos de biopsias rectales, con tinción de hematoxilina-eosina 40X. A) Tronco nervioso en submucosa, con células ganglionares maduras (expresión de un núcleo grande y excéntrico con nucléolo prominente y citoplasma abundante) señaladas con una flecha (normogangliónicos). B) Tronco nervioso hipertrófico y ausencia de células ganglionares.

catéter con un balón de $1 \times 1 \mathrm{~cm}$ en neonatos y de $3 \times 5 \mathrm{~cm}$ en lactantes y preescolares; se distiende el recto con $15 \mathrm{~mL}$ para neonatos, $30 \mathrm{~mL}$ para lactantes mayores y preescolares y $60 \mathrm{~mL}$ para niños mayores; sin embargo, pueden requerirse cantidades mayores si el paciente tiene megarrecto.

La prueba orienta al diagnóstico de enfermedad de Hirschsprung si se encuentra ausencia del reflejo anal inhibitorio. Dicho reflejo se presenta normalmente cuando un bolo de materia fecal alcanza el recto y se relaja el esfínter anal interno, es independiente de la médula espinal y está ausente cuando no existen células ganglionares inhibitorias. Debe hacerse énfasis en que se trata de una prueba de cribado/tamizaje y por lo tanto no confirma el diagnóstico. ${ }^{9}$

La expresión morfológica macroscópica se conoce como zona de transición y puede demostrarse de manera anterógrada con un colon por enema. Figura 4. En aproximadamente el 12\% de los casos, la zona de transición radiológica no corresponde con la zona de transición histopatológica, sobre todo, si la enfermedad está localizada en recto sigmoides; ${ }^{10}$ así mismo, el 


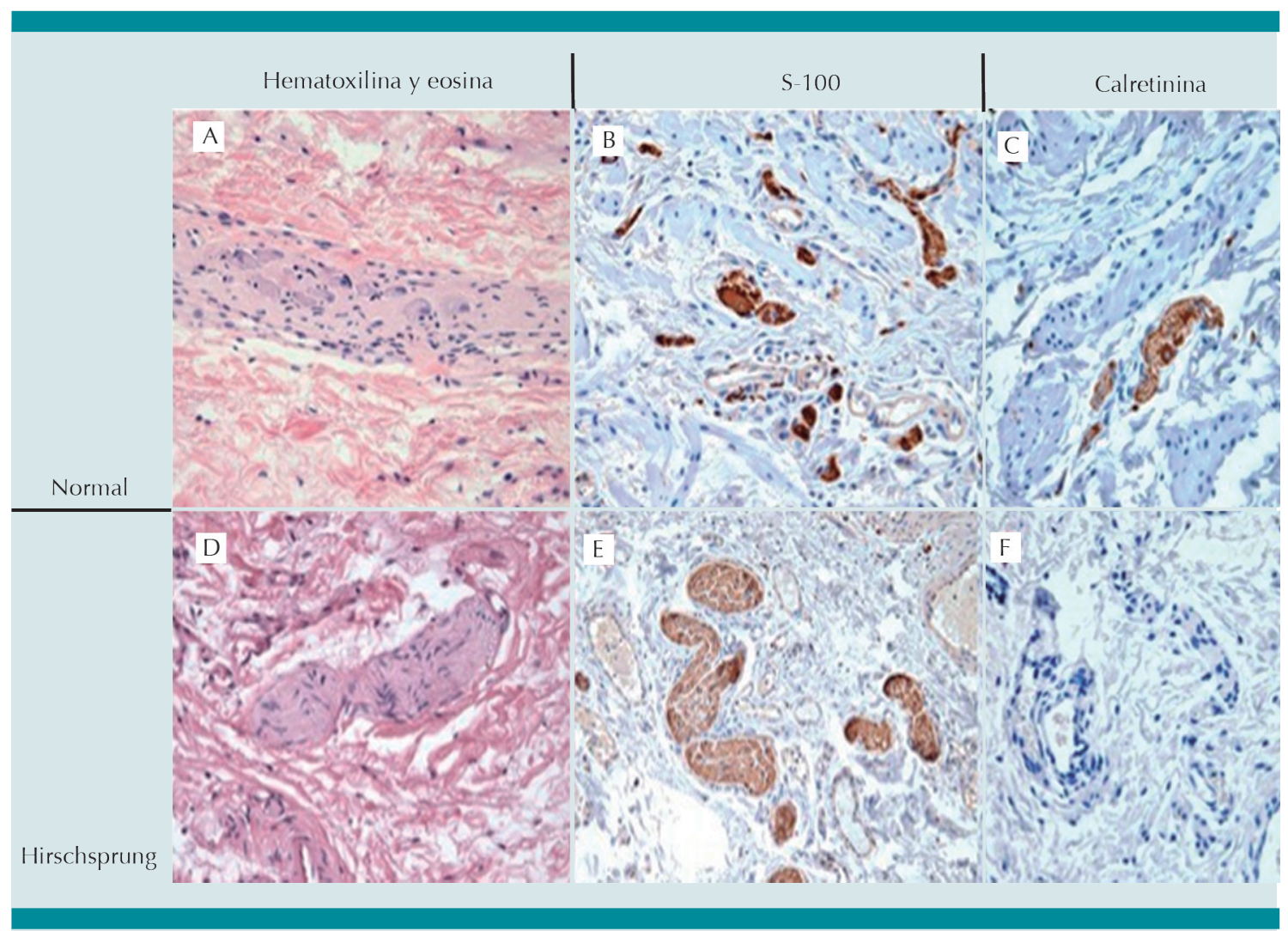

Figura 3. Cuadro comparativo de las tres tinciones en un caso normogangliónico (línea superior) y en un caso con enfermedad de Hirschsprung rectosigmoidea (línea inferior). A y D (hematoxilina y eosina); A) troncos nerviosos no hipertróficos con células ganglionares; D) Tronco nervioso hipertrófico sin células ganglionares. B y E) La inmuno histoquímica con proteína S-100 que tiñe troncos nerviosos, observándose hipertróficos en la imagen E; en C y F se observa inmunohistoquímica con calretinina; C) Revela la positividad de una pequeña célula ganglionar y neuritas y en F) la ausencia de tinción en el plexo submucoso aganliónico.

$75 \%$ de los niños con enfermedad de Hirschsprung total no muestran tal zona de transición. ${ }^{11}$

\section{TRATAMIENTO}

El objetivo global del tratamiento de la enfermedad de Hirschsprung es mejorar la calidad de vida de los pacientes. Esto se logra al obtener un número aceptable de evacuaciones fecales, conservar la continencia fecal y evitar el desarrollo de síntomas de enterocolitis. Se han descrito varias técnicas quirúrgicas para lograr estos objetivos. En 1948 Swenson estableció los tres principios para su tratamiento quirúrgico:

1. Resección del intestino enfermo (recto sigmoides en el $85 \%$ de los casos).

2. Descenso del colon "normogangliónico" en el canal anal a través de la pelvis "pullthrough".

3. Anastomosis colorrectal cerca, pero por arriba de la línea pectínea. 


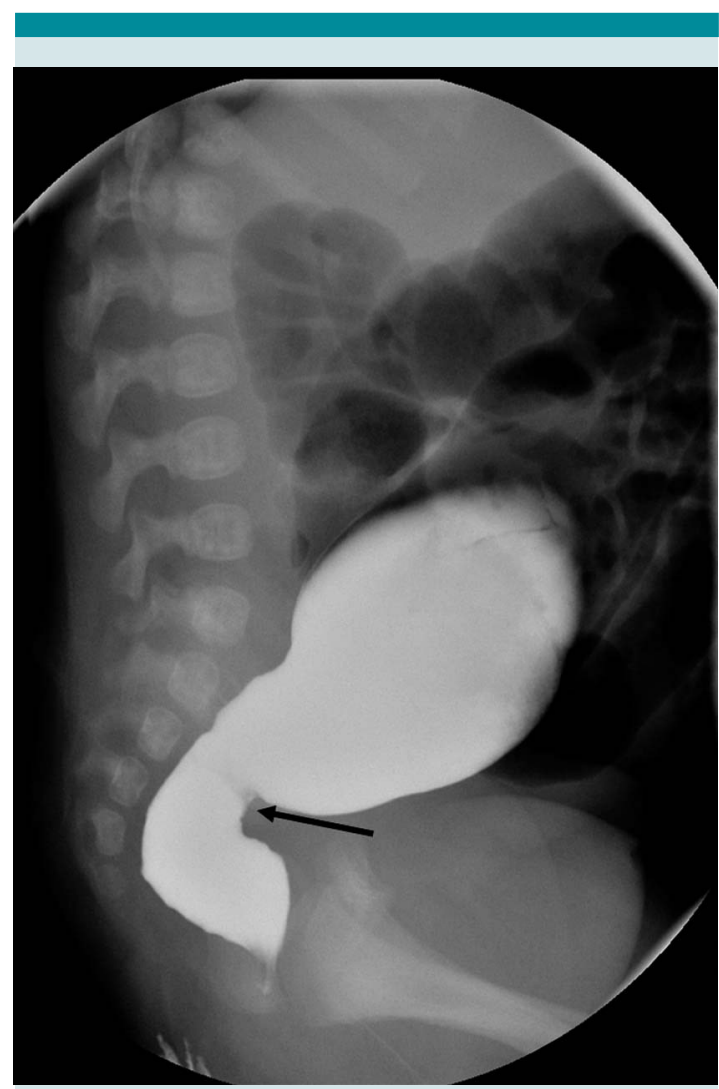

Figura 4. Colon por enema (radiografía en proyección lateral que muestra zona de transición entre el recto agangliónico y el sigmoides normogangliónico).

Esta técnica requiere destreza quirúrgica para evitar la lesión de órganos pélvicos. ${ }^{4}$

En 1956 Duhamel diseñó una cirugía que evitaba la disección pélvica anterolateral, cerrando el recto enfermo en bolsa de Hartmann a nivel de la reflexión peritoneal, resecó el colon proximal enfermo y descendió el colon normogangliónico posterior al recto, para finalmente realizar una anastomosis colorrectal termino lateral. ${ }^{4}$

Soave, en 1964, con el objetivo de evitar la disección perirrectal, describió su experiencia al descender el colon sano "normogangliónico", dentro de una funda muscular del recto nativo, al cual le reseco la mucosa y submucosa. ${ }^{4}$

La reproducción de estos procedimientos de forma abierta o laparoscópica son actualmente las modalidades terapéuticas realizadas por muchos cirujanos pediatras, mismas que pueden acompañarse o no de colostomía o ileostomía previas. Sin embargo, la necesidad de estomas actualmente es controversial; la colostomía es innecesaria en más del $70 \%$ de los recién nacidos con enfermedad de Hirschsprung limitada al recto y sigmoides. ${ }^{4}$ El uso de una sonda transrectal puede aliviar la obstrucción intestinal distal y resolver la enterocolitis a través de irrigaciones rectales; seguido de la toma de una biopsia rectal, y si ésta establece el diagnóstico de enfermedad de Hirschsprung, se realizará un estudio contrastado (colon por enema) que muestre la longitud del intestino enfermo.

Los niños pueden ser candidatos a un procedimiento de descenso transanal (sin apertura abdominal) bajo los principios de Swenson (resección de toda la pared rectal y colónica aganglionica con anastomosis colorectal) y Soave (resección de las capas mucosa y submucosa del recto, colectomía del intestino aganglionico, descenso endorrectal del colon proximal normoganglionico, dentro de un mango muscular del recto enfermo que al cual se le realiza una miotomía longitudinal posterior, que permita la apertura del cilindro rectal aganglionico, descrito por De la Torre-Ortega en 1998). ${ }^{12-14}$

En niños con abdomen agudo, con o sin datos de perforación intestinal o desequilibrio hemodinámico-hidroelectrolítico grave, una laparotomía puede ser la opción más adecuada para resolver la urgencia abdominal, si la zona de transición es evidente y se cuenta con disponibilidad de un patólogo. Una toma de biopsia por arriba de la zona de transición mostrando un intestino sano (normoganglionico), indicaría el sitio intestinal 
que se debería realizar la derivación intestinal; si se observara una perforación intestinal, lo cual ocurre más frecuentemente en el sitio dilatado intestino proximal (normoganglionico), ese sería un sitio adecuado para realizar una derivación intestinal.

Finalmente, aquellos niños en los que la obstrucción intestinal no fue resuelta con la colocación de una sonda rectal e irrigaciones, o bien se les realizó una laparotomía exploradora y la zona de transición no es evidente, son candidatos a realizar una ileostomía y toma de biopsias de espesor total para determinar la longitud del segmento intestinal enfermo. Estos son los casos de enfermedad de Hirschsprung total donde la zona de transición suele observarse en el íleon cerca de la válvula ileocecal (5-15 cm aproximadamente) a lo que se le nombra como Hirschsprung total. (Opinión del autor) . Figura 5.

\section{ENFERMEDAD DE HIRSCHSPRUNG TOTAL}

Se han descrito las técnicas de Martin, ${ }^{15}$ Duhamel, ${ }^{16}$ y Kimura $^{17}$ combinando la motilidad normal del intestino (íleon) normoganglionico, con un segmento intestinal con capacidad de absorción de agua, el cual funcionaría como un reservorio formador de heces sólidas. Desafortunadamente, los resultados han sido sub-óptimos, con estasis fecal en el intestino delgado, proliferación bacteriana, absorción de toxinas, enterocolitis, ulceración, diarrea secretora y grandes disturbios electrolíticos. ${ }^{18}$

Actualmente, lo recomendable es realizar una impecable resección del colon, descenso del íleon con una íleo-recto anastomosis $2 \mathrm{~cm}$ arriba de la línea pectina, para garantizar la preservación del mecanismo de continencia fecal. La decisión de realizar una ileostomía protectora depende de la experiencia del cirujano. Se recomienda que la funcionalidad rectal (descenso ileal sin ileostomía o cierre de ileostomía después de un descenso ileal) deba retrasarse hasta que el niño tiene entrenamiento urinario y puede sentarse en una tasa entrenadora. Cuando se preserva el canal anal, los niños iniciarán el entrenamiento fecal pocos días después, de otra manera el gran número de evacuaciones (diarrea) en un niño sin control de esfínteres generará excoriación perianal de difícil tratamiento. ${ }^{18}$

\section{COMPLICACIONES}

Existen complicaciones prevenibles como prolapso o estenosis de los estomas, dermatitis perianal, infección, abscesos y fístulas después del descenso; errores en el diagnóstico histológico (aganglionosis residual), atresias/estenosis adquiridas de la anastomosis colo-anal postoperatorias; síntomas obstructivos en descensos con reservorios (en J, o parches de rectales nativo (procedimiento de Duhamel); destrucción del canal anal e incontinencia fecal permanente y otras poco prevenibles como enterocolitis postoperatoria.

Los pacientes con enfermedad de Hirschsprung, sobre todo aquellos con aganglionosis colónica total, tienen una alta incidencia de enterocolitis postoperatoria y es imprescindible que los padres o cuidadores primarios estén entrenados para realizar irrigaciones rectales para tratarla. En caso de establecer diagnóstico de enterocolitis, debe agregarse al tratamiento médico metronidazol vía oral a dosis convencionales y, dependiendo de la gravedad, requerirá ampliación del esquema antibiótico (ampicilina y gentamicina) referido por literatura anglosajona, ayuno, apoyo de soluciones parenterales y vigilancia intrahospitalaria hasta la resolución de la enterocolitis. $^{2,18}$

Al realizar ileostomías, la complicación observada más frecuentemente es el prolapso, el cual puede complicarse con isquemia; se recomienda fijar el intestino a la pared abdominal anterior 


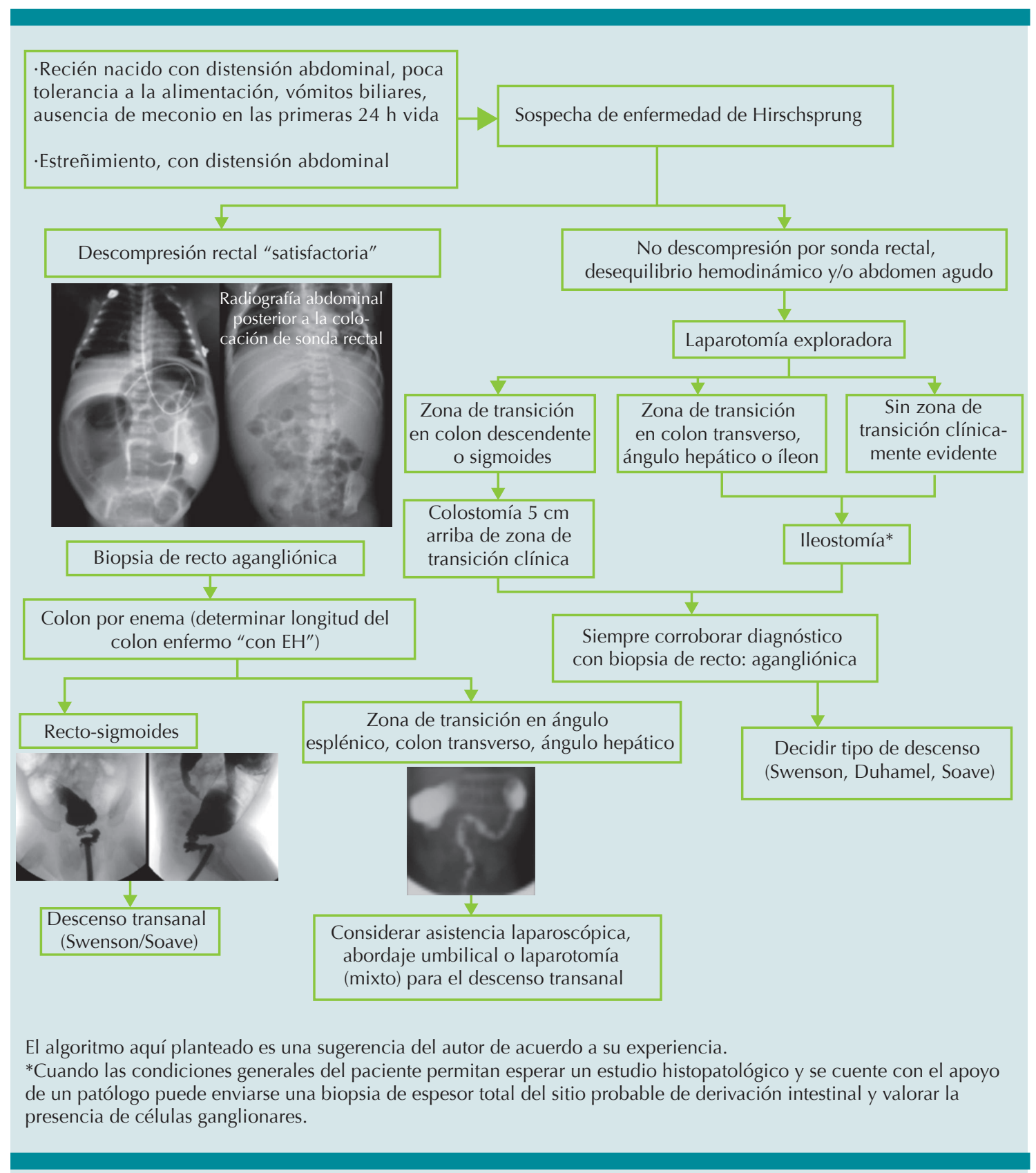

Figura 5. Algoritmo sugerido de tratamiento en la enfermedad de Hirschsprung.

aproximadamente 6-7 $\mathrm{cm}$ proximal al estoma. La segunda complicación al realizar estomas es la estenosis, se debe asegurar una adecuada irrigación arterial del intestino y una adecuada apertura de las fascias resecando un círculo de piel, aponeurosis, músculo y peritoneo, para evitar estenosis extrínseca del intestino al exteriorizarlo. 
La estenosis adquirida de la anastomosis colo-rectal se puede evitar manteniendo una irrigación arterial adecuada de las arcadas mesentéricas, del segmento intestinal descendido y evitando tensión excesiva de dicha anastomosis.

Finalmente, para no comprometer la continencia fecal se debe evitar el daño al canal anal. Para evitar esta complicación, se debe usar del retractor de Lone Star, que inicialmente permita la exposición del canal anal y gradualmente avanzar los ganchos hasta la mucosa rectal; con ello, el canal anal se encontrará protegido de la disección, la cual se deberá iniciar 1.5 a $2 \mathrm{~cm}$ por arriba de la línea dentada en la mucosa rectal. ${ }^{18}$

Debemos sospechar enfermedad de Hirschsprung en todo paciente con obstrucción intestinal distal y conocer las bases fisiopatológicas de la enterocolitis, para poder establecer tratamiento médico que permita restituir la homeostasis de los pacientes; tener un entrenamiento adecuado y suficiente exposición a este tipo de cirugía pélvicas que eviten resultados sub-óptimos, complicaciones y secuelas, y, en caso de detectarlas, implementar medidas terapéuticas adecuadas.

\section{REFERENCIAS}

1. Fitzgerald CJ. "New concepts of the etiology, diagnosis, and treatment of congenital megacolon (Hirschsprung's disease), by Orvar Swenson, MD, et al, Pediatrics, 1949;4:201209." Pediatrics 1998;102;205-7.

2. Frykman PK, Short S. Hirschsprung-associated enterocolitis: prevention and therapy. Semin Pediatr Surg. 2012;21:328-335.

3. Hirschsprung $\boldsymbol{H}$. Stuhlträgheit Neugeborner in Folge von Dilatation und Hypertrophie des Colon. Jahrbuchfür Kinderheil kundeund Physische Erziehung.1988;27:1-7.
4. De la Torre-Mondragón L. Enfermedad de Hirschsprung. Mitos y realidades a 120 años de su descripción. Acta Pediatr Mex. 2008;29(3):139-46.

5. Reyes-Múgica M. Enfermedad de Hirschsprung: neurocristopatía de la migración y la diferenciación celular. Rev Gastroenterol Mex. 1997;62(4):287-92.

6. Ridaura SC. Problemas en el diagnóstico histopatológico de la enfermedad de Hirschsprung. Acta Pediatr Mex. 2003;24(3):166-71.

7. De la Torre L, Santos K. Enfermedad de Hirschsprung: evaluación de la calretinina y S-100 como métodos complementarios en el diagnóstico de aganglionosis en biopsias rectales. Acta Pediatr Mex. 2012;33(5):246-251.

8. Lojin F, Kremer LCM, Reitsma JB, Benninga MA. Diagnostic Tests in Hirschsprung Disease: A Systematic Review. J Pediatr Gastroenterol Nutr. 2006;42(5):496-505.

9. Hong J. Clinical applications of gastrointestinal manometry in children. Pediatr Gastroenterol Hepatol Nutr. 2014;17(1):23-30.

10. De la Torre L, Langer J. Transanal Pull-Through for Hirschsprung Disease. Semin Pediatr Surg. 2010;19(2):95-106.

11. De Campo JF, Mayne V, Boldt DW, De Campo M. Radiological findings in total aganglionosis coli. Pediatr Radiol.1984;14(4):205-9.

12. De la Torre-Mondragón L, Ortega-Salgado JA. Transanalendorectal pull-through for Hirschsprung's disease. J Pediatr Surg. 1998;33:1283-6.

13. So HB, Schwartz DL, Becker JM, Daum F, Schneider KM. Endorectal pull-through without preliminary colostomy in neonates with Hirschsprung's disease. J Pediatr Surg. 1980;15(4):470-1.

14. Langer JC, Fitzgerald PG, Winthrop AL, et al. One vs two stage Soave pull-through for Hirschsprung's disease in the first year of life. J Pediatr Surg. 1996;31:33-7.

15. Martin LW. Surgical management of Hirschsprung's desease involving the small intestine. Arch Surg.1968;97(2):183-9.

16. Duhamel B. A new operation for the treatment of Hirschsprung's disease. Arch Dis Chil.1960;35:38.

17. Kimura K, Nishijima E, Muraji T, Tsugawa C, Matsumoto Y. A new surgical approach to extensive aganglionosis. J Pediatr Surg. 1981;16(6):840-3.

18. Bischoff A, Levitt MA, Peña A. Total colonic aganglionosis: a surgical challenge. How to avoid complications? Pediatr SurgInt. 2011;27:1047-52. 